\section{Bad blood}

\section{By Tracey Baas, Senior Editor}

Whereas pharmacological approaches to treating sepsis have languished in the clinic, a mechanical approach has been designed by a Massachusetts team that cleanses pathogens and endotoxins from blood. ${ }^{1}$ The microfluidic device could be a natural fit in intensive care units in which patients with severe sepsis are already being treated.

The team plans to raise funds and launch a new company to commercialize the approach.

Eighteen million cases of sepsis are diagnosed worldwide every year, with a $30 \%-50 \%$ mortality rate even within hospital intensive care units. ${ }^{2}$ No therapeutics are approved to treat sepsis, and numerous programs have failed in the clinic in part because of the complex etiology of the disease.

Standard of care includes treatment with i.v. fluids and broadspectrum antibiotics. Supportive care for patients with severe sepsis and septic shock includes administration of fluids, anti-thrombosis therapy, hemofiltration of inflammatory mediators and mechanical organ support.

Although patients with sepsis are often treated with broad-spectrum antibiotics, residual live as well as dead pathogens release toxins into the bloodstream, contributing to disease severity and mortality. ${ }^{3}$

Wyss Institute for Biologically Inspired Engineering at Harvard University founding director Donald Ingber thus set out to develop a device that could physically remove both living and dead microorganisms and endotoxins from the blood, with the goal of reducing the host immune response that drives the disease.

Ingber is also leader of the Biomimetic Microsystems platform at the Wyss Institute, a professor of vascular biology at Harvard Medical School and Boston Children's Hospital and a professor of bioengineering at the Harvard School of Engineering and Applied Sciences.

His team set out to develop a two-part system: broad-spectrum capture nanobeads to immobilize the microbes and toxins, and a microfluidic device to remove the bead-captured materials. Although Ingber's team previously used a combined micromagnetic-microfluidic technique to isolate pathogens, ${ }^{4,5}$ they never cleaned whole blood.

First, to generate nanobeads capable of capturing a broad spectrum of pathogens, the team engineered a recombinant form of a human opsonin, mannan-binding lectin (MBL2; MBL). MBL can bind to a large number of pathogenic microorganisms, including bacteria, viruses, protozoa and fungi, as well as toxins. But the protein can also activate the complement cascade and promote blood coagulation, which could complicate its use in blood. The team deleted MBL's collagen-helix domain to eliminate complement and coagulation activities while retaining pathogen binding, then modified the protein to enable uniform coating of the magnetic capture beads and simplify its manufacture.

In vitro, the magnetic MBL-modified capture nanobeads bound to a range of Gram-negative and Gram-positive bacteria, including antibiotic-resistant strains, as well as multiple fungal pathogens including Candida albicans.

Next, the team designed a spleen-inspired fluidic device that could be used to filter magnetic bead-bound pathogens from flowing blood. In the spleen, opsonized pathogens are normally cleared from blood as it seeps through adjacent endothelial cells and moves from the marginal zone and red-pulp cords into the venous sinuses of the spleen. ${ }^{6}$

To mimic this activity, the artificial biospleen device consists of a series of open rectangular slits that provide direct access between a blood channel and the saline-filled sinusoid channel (see Figure 1, "Biospleen design"). The channel and slit dimensions were chosen based on a previous fluidic device created by the group to capture and culture rare circulating tumor cells from whole blood.?

To test the efficacy of the device, the team first used the biospleen and nanobead system to remove Staphylococcus aureus spiked into saline or banked human blood, which resulted in up to $99 \%$ removal efficiency. The biospleen platform was also used to clean fresh, whole human blood

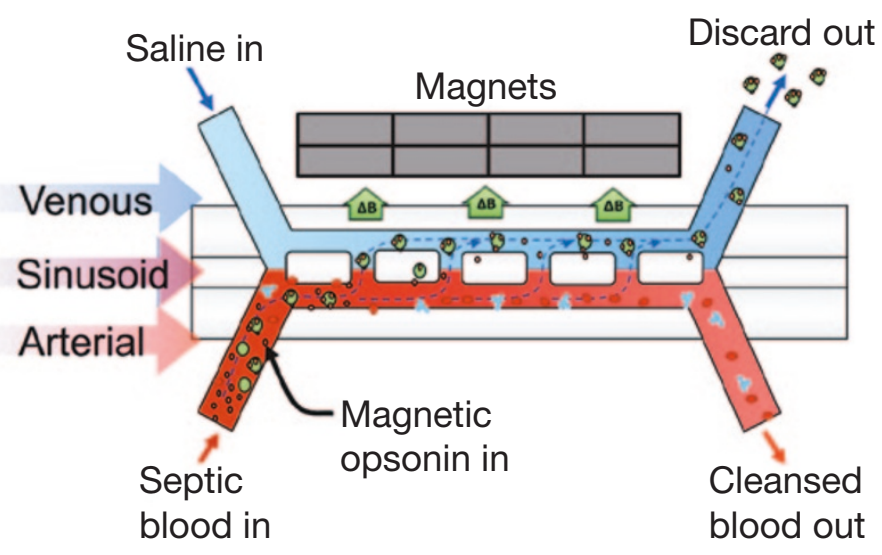

Figure 1 Biospleen design. The biospleen fluidic device incorporates a high-flow vascular arterial channel, which is perfused with septic whole blood. That channel is interconnected by open slits to a parallel low- or intermittent-flow venous sinusoid channel that is perfused with isotonic sterile saline, which mimics the architecture and function of the human spleen.

Magnetic mannan-binding lectin (MBL2; MBL)-modified nanobeads are added to the flowing septic blood and passed through an in-line spiral mixer and incubation loop, which promotes nanobeadpathogen binding before the blood enters the arterial channel of the biospleen. Stationary magnets producing a magnetic field $(\Delta \mathrm{B})$ positioned directly above the sinusoid channel pull the magnetic nanobeads and bound pathogens through the open slits, into the saline-filled channel and into a discard collection vial. (Figure adapted from Figure $1 \mathrm{c}$ in ref. 1.) 
treated with heparin, to avoid coagulation, and spiked with either $S$. aureus or lipopolysaccharide (LPS) endotoxin. To increase filtering efficiency of the small magnetic nanobeads, larger uncoated supermagnetic beads were included, which enabled the removal of up to $99.6 \%$ of all beads.

Because patients with sepsis are typically infected with multiple microorganisms, the team next tested whether the biospleen could cleanse human blood that had been spiked with rat cecal contents. The device removed $>98 \%$ of anaerobic bacteria and $>80 \%$ of aerobic bacteria after only a single pass.

Finally, the team tested the blood-cleansing capability of the biospleen system in live rat models of sepsis. They linked the microfluidic device to the jugular veins of living, anesthetized rats using medical-grade tubing and catheters.

In septic models in which rats were infected with $S$. aureus or Escherichia coli, the biospleen platform led to significantly lower levels of $S$. aureus $(p<0.01)$ or E. coli $(p<0.004)$, as well as a decrease in the levels of proinflammatory cytokines in the rats' circulation, compared with no treatment.

The procedure did not induce blood coagulation or affect the rats' temperature or breathing rates, whereas untreated septic rats showed labored breathing and lower body temperatures. In the lung, spleen and kidney, histological analyses showed lower levels of pathogen and immune cell infiltration in biospleen-treated rats than in untreated rats.

In a model of acute endotoxemic shock in which rats were injected with a lethal dose of LPS endotoxin, $89 \%$ of rats treated with the biospleen platform survived, and the system significantly decreased the amount of LPS toxin and infiltrating immune cells found in the lung, spleen and kidney compared with no treatment $(p<0.05)$. Only $14 \%$ of the untreated rats survived.

Results were published in Nature Medicine.

\section{Sepsis skepticism}

Although the biospleen system provided proof of concept in simplistic sepsis models, researchers were concerned that the multifactorial nature of sepsis would pose a significant challenge to clinical development.

Sébastien Gibot was not convinced, from a conceptual standpoint, that the biospleen would prove useful in most patients experiencing severe sepsis or septic shock because the conditions are often caused by solid organ infections, which are not addressed by the system.

"As acknowledged by the authors themselves, less than 15\%-20\% of septic shock patients present with bacteremia," noted Gibot. "As the device is only designed to clear bacteria from blood, and not infected organs, the indications are by nature limited."

Gibot is a professor of critical care medicine at the University of Lorraine Medical School in Nancy and a cofounder of Inotrem S.A., a French biotech company that specializes in the discovery and development of treatments to control immune reactions in acute inflammatory disease. The company's lead candidate is a triggering receptor expressed on myeloid cells 1 (TREM1) inhibitor that aims to control excessive inflammation in patients with severe sepsis.

He added that this is not the first time that mechanical filtration of the blood has been proposed as a therapeutic strategy. He said that removal of endotoxins or cytokines by various extracorporeal techniques of 'blood purification', such as hemofiltration with various membranes or polymyxin cartridges, has been disappointing.

"Thirty years of disappointment with sepsis-targeted treatments or devices has made me very cautious," he added. "Nevertheless, this biospleen does deserve further investigation in more relevant septic shock models."

Phillip Chan agreed that testing the device in additional model systems will be important to demonstrate clinical utility. "Ingber's team has shown strong proof of concept that the biospleen can remove a broad range of pathogens from the blood. It will be exciting to see how the biospleen platform performs in conjunction with antibiotic treatment in small- and large-animal sepsis models that simulate human infection," he noted.

Chan is CEO and president of CytoSorbents Corp., and the company works with Ingber's group and others as part of the Dialysislike Therapeutics program to treat sepsis, funded by the Defense Advanced Research Projects Agency (DARPA). Chan was not an author on the manuscript. The biospleen work was funded by DARPA.

Gibot specifically wanted to see additional sepsis models that better reflect disease in patients. "Rats with cecal ligation puncture or pneumonia, or pigs with fecal peritonitis, should be treated with the biospleen," suggested Gibot. "It would also be interesting to test the device in fully 'resuscitated' animals, where they have been treated with fluids and antibiotics before being attached to the device, to better mimic what happens in patients."

Chan added that in sepsis, timing is everything. "As with most blood purification therapies, faster intervention along with standard of care, such as antibiotics, will likely yield greater benefit," he said. "On one hand, you don't want to intervene too early with the biospleen because many patients will get better with antibiotics alone. But you also don't want to be too late because once septic shock and multiple organ dysfunction syndrome has occurred, pathogen reduction may not be sufficient and other approaches, such as cytokine reduction, will likely be needed."

CytoSorbents specializes in blood purification and markets CytoSorb as an extracorporeal cytokine adsorber in the EU and abroad. The CytoSorb cartridge works with standard hemodialysis machines and is filled with highly porous polymer beads that remove inflammatory toxins from the blood based on size exclusion and surface adsorption. The device is being used clinically to control dangerous inflammation in a broad range of life-threatening conditions, including severe sepsis and septic shock. CytoSorbents has an approved investigational device exemption (IDE) to run a small sepsis trial in the U.S. and is conducting a U.S. Air Force-funded, 30-patient human trauma trial under another FDA-approved IDE.

Chan noted that the regulatory path for the biospleen could be another hurdle to commercialization. "Unlike products that fall into neat individual categories, the FDA will most likely evaluate the biospleen as a combination biologic-device product so that, as with all such products, safety of the individual components of the system, including the recombinant mannose-binding lectin and nanoparticles, will need to be demonstrated," he said. 


\section{ANALYSIS}

Though it could take some time to finalize the development, he added that DARPA support for the project could speed the process. "The combination of DARPA support and Ingber's resourceful team will help accelerate the development process and may help get the technology to human safety trials within the next several years."

Ingber acknowledged the development challenges but noted that although only some patients with septic shock have living bacteria in their blood, most if not all have dead pathogens and toxins in their blood that elicit the inflammatory cascade. The power of the biospleen is that it cleanses the blood of these toxic materials, he said. He is also working to simplify the device design to minimize regulatory hurdles and has initiated studies in a large-animal sepsis model, but he did not disclose further details.

Multiple patent applications have been filed by Harvard University covering claims including composition of matter and methods of use related to the engineered MBL and blood-cleansing technology. Although Ingber's team is planning to form a new company around the biospleen and related technologies, the IP currently is available for licensing.

Baas, T. SciBX 7(36); doi:10.1038/scibx.2014.1062

Published online Sept. 18, 2014

\section{REFERENCES}

1. Kang, J.H. et al. Nat. Med.; published online Sept. 14, 2014; doi:10.1038/nm.3640

Contact: Donald E. Ingber, Wyss Institute for Biologically Inspired Engineering at Harvard University, Boston, Mass. e-mail: don.ingber@wyss.harvard.edu

2. Anonymous. Nat. Med. 18, 997 (2012)

3. Cohen, J. Nature 420, 885-891 (2002)

4. Xia, N. et al. Biomed. Microdevices 8, 299-308 (2006)

5. Yung, C.W. et al. Lab Chip 9, 1171-1177 (2009)

6. Mebius, R.E. \& Kraal, G. Nat. Rev. Immunol. 5, 606-616 (2005)

7. Kang, J.H. et al. Lab Chip 12, 2175-2181 (2012)

COMPANIES AND INSTITUTIONS MENTIONED

Boston Children's Hospital, Boston, Mass.

CytoSorbents Corp. (OTCBB:CTSO), Monmouth Junction, N.J. Defense Advanced Research Projects Agency, Arlington, Va. Harvard Medical School, Boston, Mass. Harvard School of Engineering and Applied Sciences, Cambridge, Mass.

Harvard University, Cambridge, Mass.

Inotrem S.A., Nancy, France

University of Lorraine Medical School in Nancy, Nancy, France Wyss Institute for Biologically Inspired Engineering at Harvard University, Boston, Mass. 